\title{
Integrated Weed Management in Coriander (Coriandrum sativum L.)
}

\author{
Shilpa Rathod, R. K. Jat, Sanjay Prajapati*, Naresh Solanki and Jay Brahmbhatt \\ Department of Fruit Science, College of Horticulture, Sardarkrushinagar Dantiwada \\ Agricultural University, Jagudan-382710, Gujarat, India \\ *Corresponding author
}

\section{Keywords}

Hand weeding, Interculturing, Pendimethalin, Oxyfluorfen,

Oxadiargyl, Pretilachlor, Quizalofop ethyl and Weed management

Article Info

\section{Accepted:}

12 April 2021

Available Online:

10 May 2021

\section{A B S T R A C T}

A field experiment was conducted during rabi season of 2019-20 at College Farm, S. D. Agricultural University, Jagudan. The treatments included of best combinations consisting of physical and chemical methods as well as alone. The studies signified the importance of hand weeding at 25 and 40 DAS or application of post emergence herbicides which could benefit the crops in reducing the different weed density and ultimately reduced the weed dry matter which resulted in increase in the crop yield. Keeping the crop weed free up to harvest recorded higher weed control efficiency $(100 \%)$ and lower weed index $(0 \%)$. Higher growth and yield attributes under physical method alone and their integration with herbicide treatments might be due to effective control of weeds, significantly reduced crop weed competition resulting in better congenial condition for growth and development of the crop. The maximum seed yield was recorded with weed free up to harvest and was at par with the treatments in which adoption of physical and chemical methods alone and their combinations. The maximum net realization and benefit cost ratio (BCR) were recorded with treatment $\mathrm{T}_{2}$ i.e. Two interculture followed by hand weed at 25 and 40 DAS.

\section{Introduction}

Coriander (Coriandrum sativum L.) is one of the most important spices crop belonging to the family apiaceae. It is commonly known as "Dhania" or "Dhana". India is known as the "Land of Spices". Spices play pivotal role in human diet as well as they give an agreeable flavor and aroma to food, which add greatly to the pleasure of eating. The stem, leaves and fruits have a pleasant aromatic odour due to the linalool containing essential oil in the fruits. The leaves are used for flavoring the curries, sauces and soups. The dried fruits are extensively used in preparation of curry powder, pickling spices, sausages and seasoning. It is also served as a flavoring agent for food preparations. Coriander is an annual herb. According to the climatic conditions, it is cultivated as a summer or winter annual 
crop. At flowering, the glabrous plant can reach height between 0.20 and $1.40 \mathrm{~m}$. The stem is more or less erect and sympodial, monochasial-branched, sometimes with several side branches at the basal node. The colour of the ribbed stem is more or less green and sometimes turns to red or violet during the flowering period. The stem can reach a diameter of up to $2 \mathrm{~cm}$. The leaves are alternate and the first ones are often gathered in a rosette. The flowers have five petals which are pink or sometimes white. The inflorescence is a compound umbel. Fruit is globular and 3 to $4 \mathrm{~mm}$ diameter, when pressed break into two locules each having one seed. Fruit has delicate fragrance; Seeds are pale and white to light brown in colour (Khan et al., 2014). Integrated weed management approach involves the use of two or more techniques selected for weed control from five general categories viz., prevention, cultural, mechanical (physical), biological and chemical in a well planned sequence so designed as not to effect the ecosystem. The integrated weed management approach is advantageous because one technique rarely achieve complete control of all weeds in a particular crop and even a relatively few surviving weeds can produce sufficient number of seeds to perpetuate the species. With the intensive farming practices, introduction of high yielding varieties, cultivation by multiple cropping and paucity of labour, the adoption of integrated approach is now a days more relevant to Indian agriculture.

\section{Materials and Methods}

The experiment consisted of twelve weed management treatments [viz., $\mathrm{T}_{1}$ : Control, $\mathrm{T}_{2}$ : Two interculture followed by hand weeding at 25 and 40 DAS, T 3 : Pendimethalin @ 1.0 $\mathrm{kg} / \mathrm{ha}$ as pre emergence, $\mathrm{T}_{4}$ : Pendimethalin@ $0.75 \mathrm{~kg} / \mathrm{ha}$ as pre emergence followed by interculture and hand weeding at 40-45 DAS,
$\mathrm{T}_{5}$ : Pendimethalin $0.5 \mathrm{~kg} / \mathrm{ha} \quad(\mathrm{PE}) \quad+$ Quizalofop ethyl $0.04 \mathrm{~kg} / \mathrm{ha}$ at $30-35 \mathrm{DAS}$, $\mathrm{T}_{6}$ : Tank mixture of Pendimethalin @ 500 g/ha + Oxyfluorfen @ 30 g/ha as pre emergence, $\mathrm{T}_{7}$ : Oxadiargyl @ $100 \mathrm{~g} / \mathrm{ha}$ as pre emergence, $\mathrm{T}_{8}$ : Oxadiargyl @ $80 \mathrm{~g} / \mathrm{ha}$ as pre emergence followed by one hand weeding at

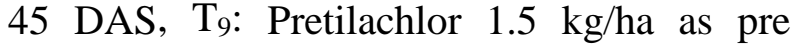
emergence, $\mathrm{T}_{10}$ : Pretilachlor $1.0 \mathrm{~kg} / \mathrm{ha}$ as pre emergence followed by interculture and hand weeding at 40-45 DAS, $\mathrm{T}_{11}$ : Pretilachlor 1.0 $\mathrm{kg} / \mathrm{ha}(\mathrm{PE})+$ Quizalofop ethyl $0.04 \mathrm{~kg} / \mathrm{ha}$ at 20 DAS and $T_{12}$ : Weed free up to harvest] were evaluated on coriander $c v$. Gujarat Coriander 3. The experiment was laid out in Randomized Block Design with three replications. Coriander variety Gujarat Coriander 3 was sown in the first week of November and $30 \mathrm{~cm}$ row to row spacing with a seed rate of $15 \mathrm{~kg} / \mathrm{ha}$. Irrigation was given immediately after sowing. All other standard cultural practices were followed during the cropping season. Pre and post emergence application of pendimethalin, oxadiargyl, oxyfluorfen, Quizalofop ethyl and Pretilachlor was done with the help of a knapsack sprayer fitted with flood fan nozzle with a spray volume of 600 1/ha. In manual weed control treatments, weeds were uprooted within the row and between the row with inter culturing as per days mentioned in each treatments. In weed free plots, the weeds were removed manually at fifteen days interval for ensuring complete weed free condition. The weed count (density) was taken from the tagged spot of $0.25 \mathrm{~m}^{2}$ in the randomly selected each net plot and were calculated and converted into square meter basis for convenience. In order to draw a valid conclusion, the weed count data were subjected to $(\sqrt{\mathrm{X}+0.5})$ as suggested by Gomez and Gomez (1984) before statistical analysis. For dry weight of weeds, the weeds were air dried completely till they attained constant weight and finally recorded for each treatment after harvest and converted in to $\mathrm{kg} / \mathrm{ha}$. Weed control efficiency and weed 
index were calculated by the formulae suggested by Kondap \& Upadhyay (1985) and Gill \& Kumar (1969). Statistical analysis procedure was followed as suggested by Panse $\&$ Sukhatme (1985). The observations were recorded on dry weight of weeds, weed control efficiency, weed index, seed yield and straw yield by adopting appropriate procedure.

\section{Results and Discussion}

\section{Growth and yield attributes}

Growth and yield attributes of coriander were significantly influenced by different weed management treatments (Table 1). All growth and yield attributing characters viz., plant population, plant height, numbers of branches/plant, numbers of umbels/plant, number of umbellates/umbel, number of seeds/umbellate were significantly maximum under weed free crop condition. Higher growth and yield attributes under physical method alone and their integration with herbicide treatments might be due to effective control of weeds, significantly reduced crop weed competition ultimately resulting in better congenial condition for growth and development of the crop. However these values were minimum under control condition. These findings are also in conformity with those reported by Patil et al., (2020) in coriander, Meena et al., (2009), Meena and Mehta (2010), Yadav et al., (2012) and Birla et al., (2016) in cumin, Meena and Mehta (2009) in fennel, Meena et al., (2015) and Patel et al., (2019) in ajwain.

\section{Seed and straw yield}

Seed and straw yield of coriander were significantly influenced by different weed management treatments during the course of investigation (Table 2). The maximum seed yield (1634 kg/ha) was recorded under treatment weed free up to harvest $\left(\mathrm{T}_{12}\right)$, which was statistically at par with treatments $T_{2}, T_{4}$, $\mathrm{T}_{5}, \mathrm{~T}_{3}$ and $\mathrm{T}_{6}$. The maximum straw yield (2242 $\mathrm{kg} / \mathrm{ha}$ )) was recorded in treatment weed free up to harvest, which was found statistically at par with all the treatments except $\mathrm{T}_{1}, \mathrm{~T}_{9}, \mathrm{~T}_{10}$ and $\mathrm{T}_{11}$. However, significantly minimum seed and straw yield was recorded when weed management practices were not adopted i.e. control $\left(\mathrm{T}_{1}\right)$. Alone Pretilachlor $1.5 \mathrm{~kg} / \mathrm{ha}$ as pre emergence or Pretilachlor $1.0 \mathrm{~kg} / \mathrm{ha}(\mathrm{PE})+$ Quizalofop ethyl $0.04 \mathrm{~kg} / \mathrm{ha}$ at $20 \mathrm{DAS}$ application of these two herbicides were not found to be effective to control the weeds in coriander. Removal of weeds throughout crop growth period by physical and integrated weed management practices provide better space and resource to the crop plants (light, water, nutrient, space, etc.), which ultimately favoured better environment for growth and development of the plant which might be increased yield attributes. Analoguous findings have been reported by Patil et al., (2020) in coriander, Mehriya et al., (2008), Yadav et al., (2012) and Birla et al., (2016) in cumin, Meena et al., (2013) in dill seed, Meena and Mehta (2009) in fennel, Meena et al., (2015) and Patel et al., (2019) in ajwain.

\section{Quality parametres}

The essential oil content and test weight as influenced by different weed management treatments recorded after harvest (Table 2). A perusal of data showed that different weed management treatments exhibited their non significance. The maximum test weight (14.26 g) was produced by treatment weed free up to harvest $\left(\mathrm{T}_{12}\right)$, which was found statistically at par with all treatments except $\mathrm{T}_{1}, \mathrm{~T}_{7}, \mathrm{~T}_{9} \mathrm{~T}_{10}$ and $\mathrm{T}_{11}$. Complete elimination of weeds from the field since the beginning which give the chance to the crop to grow under weed free condition. This condition ultimately resulted in better utilization of nutrients and moisture available in the soil by the crop and might have produced bold seeds. 
Table.1 Growth and yield attributes of coriander as influenced by different weed management treatments.

\begin{tabular}{|c|c|c|c|c|c|c|c|c|}
\hline \multirow[t]{2}{*}{ Treatments } & \multicolumn{2}{|c|}{$\begin{array}{c}\text { Plant } \\
\text { population/net plot }\end{array}$} & \multicolumn{2}{|c|}{$\begin{array}{l}\text { Plant height } \\
(\mathrm{cm})\end{array}$} & \multirow{2}{*}{$\begin{array}{l}\text { No. of } \\
\text { branches/ } \\
\text { plant }\end{array}$} & \multirow{2}{*}{$\begin{array}{l}\text { No. of } \\
\text { umbels/ } \\
\text { plant }\end{array}$} & \multirow{2}{*}{$\begin{array}{l}\text { No. of } \\
\text { umbellates/ } \\
\text { umbel }\end{array}$} & \multirow{2}{*}{$\begin{array}{l}\text { No. of } \\
\text { seeds/ } \\
\text { umbellate }\end{array}$} \\
\hline & 20 DAS & $\begin{array}{c}\text { At } \\
\text { harvest }\end{array}$ & 30 DAS & $\begin{array}{c}\text { At } \\
\text { harvest }\end{array}$ & & & & \\
\hline $\mathbf{T}_{1}:$ Control & 276 & 273 & 8.26 & 83.32 & 4.80 & 16.25 & 4.18 & 4.83 \\
\hline $\begin{array}{l}T_{2}: \text { Two interculture followed by hand weeding } \\
\text { at } 25 \text { and } 40 \text { DAS }\end{array}$ & 306 & 301 & 11.93 & 101.95 & 7.11 & 24.78 & 6.14 & 7.11 \\
\hline $\begin{array}{l}\mathbf{T}_{3}: \text { Pendimethalin } @ 1.0 \quad \mathrm{~kg} / \mathrm{ha} \text { as } \text { pre } \\
\text { emergence }\end{array}$ & 299 & 295 & 11.51 & 99.73 & 6.82 & 23.70 & 5.82 & 6.91 \\
\hline $\begin{array}{l}T_{4}: \text { Pendimethalin @ } 0.75 \mathrm{~kg} / \mathrm{ha} \text { as pre } \\
\text { emergence followed by interculture and hand } \\
\text { weeding at } 40-45 \text { DAS }\end{array}$ & 302 & 297 & 11.82 & 101.48 & 7.02 & 24.44 & 6.06 & 7.04 \\
\hline $\begin{array}{l}T_{5}: \text { Pendimethalin } 0.5 \mathrm{~kg} / \mathrm{ha}(\mathrm{PE})+\text { Quizalofop } \\
\text { ethyl } 0.04 \mathrm{~kg} / \mathrm{ha} \text { at } 30-35 \mathrm{DAS}\end{array}$ & 301 & 298 & 11.73 & 100.05 & 7.02 & 24.09 & 6.06 & 7.00 \\
\hline $\begin{array}{l}\mathrm{T}_{6} \text { : Tank mixture of Pendimethalin @ } 500 \mathrm{~g} / \mathrm{ha} \\
\text { + Oxyfluorfen @ } 30 \mathrm{~g} / \mathrm{ha} \text { aspre emergence }\end{array}$ & 299 & 295 & 11.31 & 99.16 & 6.74 & 23.02 & 5.80 & 6.84 \\
\hline$T_{7}:$ Oxadiargyl @ 100 g/ha as pre emergence & 296 & 292 & 10.92 & 97.58 & 6.40 & 22.64 & 5.75 & 6.02 \\
\hline $\begin{array}{l}\mathrm{T}_{8} \text { :Oxadiargyl @ } 80 \mathrm{~g} / \mathrm{ha} \text { as pre emergence } \\
\text { followed by one hand weeding at } 45 \mathrm{DAS}\end{array}$ & 297 & 295 & 11.19 & 98.67 & 6.45 & 23.37 & 5.92 & 6.64 \\
\hline $\mathrm{T}_{9}:$ Pretilachlor $1.5 \mathrm{~kg} / \mathrm{ha}$ as pre emergence & 292 & 289 & 9.82 & 94.04 & 6.13 & 20.88 & 5.46 & 5.10 \\
\hline $\begin{array}{l}T_{10} \text { : Pretilachlor } 1.0 \mathrm{~kg} / \mathrm{ha} \text { as pre emergence } \\
\text { followed by interculture and hand weeding at } \\
40-45 \mathrm{DAS}\end{array}$ & 294 & 291 & 10.50 & 97.99 & 6.27 & 22.07 & 5.71 & 6.47 \\
\hline $\begin{array}{l}\text { T11: Pretilachlor } 1.0 \mathrm{~kg} / \mathrm{ha}(\mathrm{PE})+\text { Quizalofop } \\
\text { ethyl } 0.04 \mathrm{~kg} / \mathrm{ha} \text { at } 20 \mathrm{DAS}\end{array}$ & 293 & 289 & 10.48 & 96.74 & 6.13 & 21.48 & 5.66 & 6.22 \\
\hline$T_{12}:$ Weed free up to harvest & 307 & 303 & 12.75 & 103.55 & 7.20 & 25.58 & 6.23 & 7.15 \\
\hline S.Em. \pm & 6.23 & 5.43 & 0.51 & 3.22 & 0.23 & 0.82 & 0.25 & 0.25 \\
\hline C.D. at $5 \%$ & NS & NS & 1.50 & 9.43 & 0.68 & 2.40 & 0.74 & 0.72 \\
\hline C.V. \% & 3.63 & 3.21 & 8.06 & 5.69 & 6.20 & 6.25 & 7.62 & 6.60 \\
\hline
\end{tabular}


Table.2 Yield and Quality parameters of coriander as influenced by different weed management treatments.

\begin{tabular}{|c|c|c|c|c|}
\hline Treatments & $\begin{array}{l}\text { Seed yield } \\
(\mathrm{kg} / \mathrm{ha})\end{array}$ & $\begin{array}{l}\text { Straw yield } \\
\quad \text { (kg/ha) }\end{array}$ & $\begin{array}{c}\text { Essential oil } \\
\text { content } \\
(\%)\end{array}$ & $\begin{array}{l}\text { Test weight } \\
\text { (g) }\end{array}$ \\
\hline $\mathbf{T}_{1}:$ Control & 1128 & 1733 & 0.35 & 10.32 \\
\hline$T_{2}$ : Two interculture followed by hand weeding at 25 and 40 DAS & 1588 & 2191 & 0.44 & 14.05 \\
\hline$T_{3}:$ Pendimethalin @ $1.0 \mathrm{~kg} / \mathrm{ha}$ as pre emergence & 1486 & 2089 & 0.41 & 13.00 \\
\hline $\begin{array}{l}T_{4}: \text { Pendimethalin @ } 0.75 \mathrm{~kg} / \mathrm{ha} \text { as pre emergence followed by interculture and } \\
\text { hand weeding at 40-45 DAS }\end{array}$ & 1546 & 2150 & 0.42 & 13.82 \\
\hline$T_{5}:$ Pendimethalin $0.5 \mathrm{~kg} / \mathrm{ha}(\mathrm{PE})+$ Quizalofop ethyl $0.04 \mathrm{~kg} / \mathrm{ha}$ at $30-35$ DAS & 1501 & 2103 & 0.41 & 13.40 \\
\hline $\begin{array}{l}\mathrm{T}_{6} \text { : Tank mixture of Pendimethalin @ } 500 \mathrm{~g} / \mathrm{ha}+\text { Oxyfluorfen @ } 30 \mathrm{~g} / \mathrm{ha} \text { aspre } \\
\text { emergence }\end{array}$ & 1465 & 2068 & 0.40 & 13.21 \\
\hline$T_{7}$ :Oxadiargyl @ 100 g/ha as pre emergence & 1406 & 2012 & 0.37 & 12.36 \\
\hline $\begin{array}{l}T_{8}: \text { Oxadiargyl @ } 80 \mathrm{~g} / \mathrm{ha} \text { as pre emergence followed by one hand weeding at } 45 \\
\text { DAS }\end{array}$ & 1428 & 2031 & 0.38 & 13.00 \\
\hline $\mathrm{T}_{9}$ : Pretilachlor $1.5 \mathrm{~kg} / \mathrm{ha}$ as pre emergence & 1206 & 1811 & 0.35 & 12.05 \\
\hline $\begin{array}{l}T_{10} \text { : Pretilachlor } 1.0 \mathrm{~kg} / \mathrm{ha} \text { as pre emergence followed by interculture and hand } \\
\text { weeding at } 40-45 \text { DAS }\end{array}$ & 1364 & 1967 & 0.37 & 13.20 \\
\hline$T_{11}$ : Pretilachlor $1.0 \mathrm{~kg} / \mathrm{ha}(\mathrm{PE})+$ Quizalofop ethyl $0.04 \mathrm{~kg} / \mathrm{ha}$ at 20 DAS & 1278 & 1883 & 0.36 & 12.40 \\
\hline$T_{12}:$ Weed free up to harvest & 1634 & 2242 & 0.44 & 14.26 \\
\hline S.Em. \pm & 67.74 & 91.31 & 0.03 & 0.46 \\
\hline C.D. at $5 \%$ & 198.64 & 267.78 & NS & 1.34 \\
\hline C.V. \% & 8.27 & 7.82 & 13.67 & 6.13 \\
\hline
\end{tabular}


Table.3 Weed count (numbers $/ \mathrm{m}^{2}$ ) in coriander crop at 30, 60 DAS and at harvest as influenced by different weed management treatments

\begin{tabular}{|c|c|c|c|c|c|c|c|c|c|c|c|c|}
\hline \multirow{2}{*}{ Treatments } & \multicolumn{3}{|c|}{ Sedge } & \multicolumn{3}{|c|}{ Monocot } & \multicolumn{3}{|c|}{ Dicot } & \multicolumn{3}{|c|}{ Total } \\
\hline & $\begin{array}{c}30 \\
\text { DAS }\end{array}$ & $\begin{array}{c}\text { 60 } \\
\text { DAS }\end{array}$ & $\begin{array}{c}\text { At } \\
\text { harvest }\end{array}$ & $\begin{array}{c}\text { 30 } \\
\text { DAS }\end{array}$ & $\begin{array}{c}\text { 60 } \\
\text { DAS }\end{array}$ & $\begin{array}{c}\text { At } \\
\text { harvest }\end{array}$ & $\begin{array}{c}30 \\
\text { DAS }\end{array}$ & $\begin{array}{c}\text { 60 } \\
\text { DAS }\end{array}$ & $\begin{array}{c}\text { At } \\
\text { harvest }\end{array}$ & $\begin{array}{c}30 \\
\text { DAS }\end{array}$ & $\begin{array}{c}\text { 60 } \\
\text { DAS }\end{array}$ & $\begin{array}{c}\text { At } \\
\text { harvest }\end{array}$ \\
\hline $\mathrm{T}_{1}:$ Control & 2.68 & 3.19 & 3.29 & 3.19 & 3.89 & 4.02 & 3.54 & 4.34 & 4.42 & 5.36 & 6.57 & 6.75 \\
\hline $\begin{array}{l}T_{2}: \text { Two interculture followed by hand weeding at } \\
25 \text { and } 40 \text { DAS }\end{array}$ & 1.35 & 1.35 & 1.58 & 1.47 & 1.78 & 2.04 & 1.78 & 2.12 & 2.42 & 2.73 & 2.92 & 3.39 \\
\hline$T_{3}$ : Pendimethalin @ 1.0 kg/ha as pre emergence & 1.87 & 2.27 & 2.48 & 1.87 & 3.14 & 3.24 & 1.96 & 2.80 & 2.97 & 3.47 & 4.67 & 4.95 \\
\hline $\begin{array}{l}T_{4}: \text { Pendimethalin @ } 0.75 \mathrm{~kg} / \mathrm{ha} \text { as pre emergence } \\
\text { followed by interculture and hand weeding at } 40-45 \\
\text { DAS }\end{array}$ & 1.96 & 1.58 & 1.78 & 2.12 & 1.87 & 2.27 & 1.96 & 2.48 & 2.61 & 3.32 & 3.34 & 3.76 \\
\hline $\begin{array}{l}T_{5}: \text { Pendimethalin } 0.5 \mathrm{~kg} / \mathrm{ha}(\mathrm{PE})+\text { Quizalofop } \\
\text { ethyl } 0.04 \mathrm{~kg} / \mathrm{ha} \text { at 30-35 DAS }\end{array}$ & 2.12 & 2.12 & 2.42 & 2.27 & 2.55 & 2.74 & 2.12 & 2.61 & 2.86 & 3.57 & 4.10 & 4.53 \\
\hline $\begin{array}{l}\mathrm{T}_{6} \text { : Tank mixture of Pendimethalin @ } 500 \mathrm{~g} / \mathrm{ha}+ \\
\text { Oxyfluorfen @ 30g/ha as pre emergence }\end{array}$ & 2.20 & 2.61 & 2.74 & 2.42 & 3.19 & 3.29 & 2.20 & 3.14 & 3.24 & 3.78 & 5.08 & 5.28 \\
\hline$T_{7}:$ Oxadiargyl @ 100 g/ha as pre emergence & 2.35 & 2.86 & 2.97 & 2.55 & 3.76 & 3.85 & 2.27 & 3.67 & 3.85 & 4.33 & 5.90 & 6.12 \\
\hline $\begin{array}{l}\mathrm{T}_{8} \text { :Oxadiargyl @ } 80 \mathrm{~g} / \mathrm{ha} \text { as pre emergence } \\
\text { followed by one hand weeding at } 45 \mathrm{DAS}\end{array}$ & 2.48 & 1.47 & 1.68 & 2.80 & 1.78 & 2.20 & 2.42 & 2.42 & 2.68 & 4.28 & 3.19 & 3.72 \\
\hline $\mathrm{T}_{9}:$ Pretilachlor $1.5 \mathrm{~kg} / \mathrm{ha}$ as pre emergence & 2.55 & 2.97 & 3.19 & 3.08 & 3.81 & 3.89 & 2.86 & 3.89 & 4.02 & 4.65 & 6.12 & 6.36 \\
\hline $\begin{array}{l}T_{10}: \text { Pretilachlor } 1.0 \mathrm{~kg} / \mathrm{ha} \text { as pre emergence } \\
\text { followed by interculture and hand weeding at } 40-45 \\
\text { DAS }\end{array}$ & 2.68 & 1.68 & 1.78 & 3.14 & 2.27 & 2.48 & 2.97 & 3.08 & 3.19 & 4.71 & 4.06 & 4.30 \\
\hline $\begin{array}{l}T_{11}: \text { Pretilachlor } 1.0 \mathrm{~kg} / \mathrm{ha}(\mathrm{PE})+\text { Quizalofop ethyl } \\
0.04 \mathrm{~kg} / \mathrm{ha} \text { at } 20 \text { DAS }\end{array}$ & 2.20 & 2.61 & 2.80 & 2.42 & 3.14 & 3.34 & 2.12 & 3.29 & 3.39 & 3.75 & 5.15 & 5.43 \\
\hline$T_{12}:$ Weed free up to harvest & 0.71 & 0.71 & 0.71 & 0.71 & 0.71 & 0.71 & 0.71 & 0.71 & 0.71 & 0.71 & 0.71 & 0.71 \\
\hline S.Em. \pm & 0.14 & 0.12 & 0.11 & 0.11 & 0.13 & 0.14 & 0.14 & 0.16 & 0.13 & 0.17 & 0.22 & 0.18 \\
\hline C.D. at $5 \%$ & 0.40 & 0.34 & 0.34 & 0.33 & 0.39 & 0.42 & 0.42 & 0.46 & 0.39 & 0.49 & 0.63 & 0.53 \\
\hline C.V. \% & 11.42 & 9.43 & 8.70 & 8.49 & 8.66 & 8.83 & 11.11 & 9.47 & 7.57 & 7.83 & 8.66 & 6.76 \\
\hline
\end{tabular}


Table.4 Dry weight of weeds and Weed indices of coriander as influenced by different weed management treatments.

\begin{tabular}{|c|c|c|c|}
\hline Treatments & $\begin{array}{c}\text { Dry weight } \\
\text { of weeds } \\
\text { (kg/ha) }\end{array}$ & $\begin{array}{c}\text { Weed control } \\
\text { efficiency } \\
(\%)\end{array}$ & $\begin{array}{c}\text { Weed } \\
\text { index } \\
(\%)\end{array}$ \\
\hline $\mathbf{T}_{1}:$ Control & 917 & 0.00 & 31.10 \\
\hline$T_{2}$ : Two interculture followed by hand weeding at 25 and 40 DAS & 113 & 87.89 & 2.82 \\
\hline$T_{3}$ : Pendimethalin @ $1.0 \mathrm{~kg} / \mathrm{ha}$ as pre emergence & 231 & 74.78 & 9.02 \\
\hline $\begin{array}{l}T_{4}: \text { Pendimethalin @ } 0.75 \mathrm{~kg} / \mathrm{ha} \text { as pre emergence followed by interculture and hand } \\
\text { weeding at 40-45 DAS }\end{array}$ & 139 & 85.26 & 5.38 \\
\hline$T_{5}:$ Pendimethalin $0.5 \mathrm{~kg} / \mathrm{ha}(\mathrm{PE})+$ Quizalofop ethyl $0.04 \mathrm{~kg} / \mathrm{ha}$ at $30-35$ DAS & 204 & 77.61 & 8.21 \\
\hline $\begin{array}{l}T_{6}: \text { Tank mixture of Pendimethalin @ } 500 \text { g/ha + Oxyfluorfen @ } 30 \text { g/ha aspre } \\
\text { emergence }\end{array}$ & 312 & 65.84 & 10.38 \\
\hline $\mathrm{T}_{7}:$ Oxadiargyl @ $100 \mathrm{~g} / \mathrm{ha}$ as pre emergence & 453 & 49.90 & 13.88 \\
\hline$T_{8}$ :Oxadiargyl @ $80 \mathrm{~g} / \mathrm{ha}$ as pre emergence followed by one hand weeding at 45 DAS & 175 & 80.76 & 12.47 \\
\hline T9: Pretilachlor $1.5 \mathrm{~kg} / \mathrm{ha}$ as pre emergence & 523 & 42.22 & 26.24 \\
\hline $\begin{array}{l}T_{10} \text { : Pretilachlor } 1.0 \mathrm{~kg} / \mathrm{ha} \text { as pre emergence followed by interculture and hand weeding } \\
\text { at40-45 DAS }\end{array}$ & 196 & 78.86 & 16.31 \\
\hline$T_{11}$ : Pretilachlor $1.0 \mathrm{~kg} / \mathrm{ha}(\mathrm{PE})+$ Quizalofop ethyl $0.04 \mathrm{~kg} / \mathrm{ha}$ at $20 \mathrm{DAS}$ & 487 & 46.63 & 21.70 \\
\hline$T_{12}:$ Weed free up to harvest & 0 & 100 & 0.00 \\
\hline S.Em. \pm & 41.17 & - & - \\
\hline C.D. at $5 \%$ & 120.72 & - & - \\
\hline C.V. $\%$ & 22.82 & - & - \\
\hline
\end{tabular}


Table.5 Economics of coriander as influenced by different weed management treatments.

\begin{tabular}{|c|c|c|c|c|c|c|}
\hline Treatments & $\begin{array}{c}\text { Seed } \\
\text { yield } \\
\text { (kg/ha) }\end{array}$ & $\begin{array}{c}\text { Straw } \\
\text { yield } \\
(\mathrm{kg} / \mathrm{ha})\end{array}$ & $\begin{array}{c}\text { Gross } \\
\text { returns } \\
(₹=/ h a)\end{array}$ & $\begin{array}{c}\text { Cost of } \\
\text { cultivation } \\
\text { (₹/ha) }\end{array}$ & $\begin{array}{c}\text { Net } \\
\text { returns } \\
\text { (₹/ha) }\end{array}$ & BCR \\
\hline $\mathbf{T}_{1}:$ Control & 1128 & 1733 & 106692 & 52550 & 54142 & 2.03 \\
\hline $\begin{array}{l}T_{2}: \text { Two interculture followed by hand weeding at } 25 \text { and } 40 \\
\text { DAS }\end{array}$ & 1588 & 2191 & 149493 & 56710 & 92783 & 2.64 \\
\hline$T_{3}:$ Pendimethalin @ $1.0 \mathrm{~kg} / \mathrm{ha}$ as pre emergence & 1486 & 2089 & 139980 & 54625 & 85355 & 2.56 \\
\hline $\begin{array}{l}T_{4}: \text { Pendimethalin @ } 0.75 \mathrm{~kg} / \mathrm{ha} \text { as pre emergence followed by } \\
\text { interculture and hand weeding at } 40-45 \mathrm{DAS}\end{array}$ & 1546 & 2150 & 145563 & 56871 & 88692 & 2.56 \\
\hline $\begin{array}{l}T_{5}: \text { Pendimethalin } 0.5 \mathrm{~kg} / \mathrm{ha}(\mathrm{PE})+\text { Quizalofop ethyl } 0.04 \mathrm{~kg} / \mathrm{ha} \\
\text { at } 30-35 \text { DAS }\end{array}$ & 1501 & 2103 & 141372 & 55778 & 85594 & 2.53 \\
\hline $\begin{array}{l}\text { T }_{6} \text { : Tank mixture of Pendimethalin @ } 500 \text { g/ha + Oxyfluorfen @ } \\
30 \text { g/ha as pre emergence }\end{array}$ & 1465 & 2068 & 138027 & 54140 & 83887 & 2.55 \\
\hline$T_{7}$ :Oxadiargyl @100 g/ha as pre emergence & 1406 & 2012 & 132549 & 54376 & 78173 & 2.44 \\
\hline $\begin{array}{l}T_{8}: \text { Oxadiargyl @ } 80 \mathrm{~g} / \mathrm{ha} \text { as pre emergence followed by one hand } \\
\text { weeding at } 45 \text { DAS }\end{array}$ & 1428 & 2031 & 134586 & 56220 & 78366 & 2.39 \\
\hline $\mathrm{T}_{9}:$ Pretilachlor $1.5 \mathrm{~kg} / \mathrm{ha}$ as pre emergence & 1206 & 1811 & 113946 & 54848 & 59098 & 2.08 \\
\hline $\begin{array}{l}\mathrm{T}_{10}: \text { Pretilachlor } 1.0 \mathrm{~kg} / \mathrm{ha} \text { as pre emergence followed by } \\
\text { interculture and hand weeding at } 40-45 \mathrm{DAS}\end{array}$ & 1364 & 1967 & 128634 & 56898 & 71736 & 2.26 \\
\hline 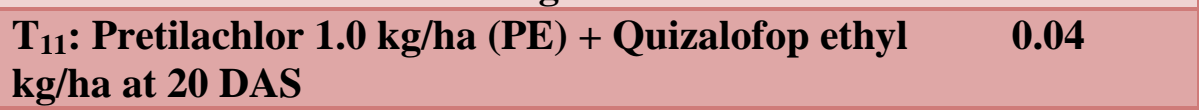 & 1278 & 1883 & 120642 & 56160 & 64482 & 2.15 \\
\hline$T_{12}$ : Weed free up to harvest & 1634 & 2242 & 153759 & 62950 & 90809 & 2.44 \\
\hline
\end{tabular}

Price- Coriander seed -140/kg, Pendimethalin-390/lit, Oxadiargyl-9420/kg, Oxyfluorfen-1600/lit, Pretilachlor-500/lit, Quizalofop ethyl-1380/lit Selling Price- Coriander seed-90/kg, Coriander straw-3/kg 
This result was supported by Mehriya et al., (2008), Meena et al., (2009), Yadav et al., (2012) and Birla et al., (2016) in cumin, Meena et al., (2015) and Patel et al., (2019) in ajwain.

\section{Weed Count}

Significantly maximum total weed count was recorded under control condition where as it was significantly lowest with treatments $T_{12}$ and $\mathrm{T}_{2}$ at 30 DAS, 60 DAS and at harvest (Table 3). Various herbicides were equally effective the controlling sedges and monocot weeds. The studies signified the importance of hand weeding at 25 and 40 DAS or application of post emergence herbicides which could benefit the crops by reducing the different weeds which ultimately reduced the weeding frequency during crop weed competition period of crop. Effective control of weeds either by manual weeding or herbicides or integrated approach. The removal of weeds at regular interval through hand weeding accounted for less count of total density of weeds. These findings are in concurrence with those of Sagarka et al., (2005) and Patil et al., (2020) in coriander, Birla et al., (2016) in cumin and Patel et al., (2019) in ajwain.

\section{Dry weight of weeds}

Significantly the highest weed dry matter was recorded with control at harvest shows in Table 4. The studies suggested the importance of hand weeding at 25 and 40 DAS or application of post emergence herbicides which could benefit the crops by reducing the weed dry matter and ultimately increasing the crop yield. These findings are in concurrence with those of Meena and Mehta (2010) and Patil et al., (2020) in coriander, Meena et al., (2009) in cumin, Meena and Mehta (2009) in fennel, Meena et al., (2013) in dill seed, Meena et al., (2015) and Patel et al., (2019) in ajwain.

\section{Weed control efficiency and Weed index}

Keeping the crop weed free up to harvest recorded higher weed control efficiency (100 $\%)$ and lower weed index $(0 \%)$ values followed by two interculture followed hand weeding at $25 \& 40$ DAS (87.89\% and 2.82 $\%$ ) and application of Pendimethalin @ 0.75 $\mathrm{kg} / \mathrm{ha}$ as pre emergence followed by interculture and hand weeding at 40-45 DAS $(85.26 \%$ and $5.38 \%)$, respectively. Elimination of weeds by manual weeding and by herbicides. The combined effect on dry weight of weeds and seed yield might have been responsible for excellent weed indices. These findings are report of Patil et al., (2020) in coriander, Mehriya et al., (2008), Meena et al., (2009) and Meena and Mehta (2010) in cumin, Meena and Mehta (2009) in fennel, Meena et al., (2013) in dill seed, Meena et al., (2015) and Patel et al. (2019) in ajwain.

\section{Economics}

The maximum seed yield, gross realization and cost of cultivation were recorded under treatment $\mathrm{T}_{12}$ :Weed free up to harvest (Table $5)$, whereas, the maximum net realization and benefit cost ratio (BCR) were recorded with treatment $\mathrm{T}_{2}$ i.e. Two interculture followed by hand weeding at 25 and 40 DAS. The higher seed yield under these treatments as a result of better weed control is responsible for higher net realization per hectar.

Based on the results of the experiment, it was concluded that weed free up to harvest in coriander is beneficial for obtaining maximum seed and straw yield. Two interculture followed by hand weeding at 25 and 40 DAS is beneficial for higher economic returns. However, application of pendimethalin@1.0 kg/ha as pre emergence 
may also be employed for weed management in coriander under scarcity of labour.

\section{References}

Birla, L.; Naruka, I. S.; Shaktawat, R. P. and Ajnave, S. R. (2016). Integrated weed management in cumin. Indian Journal of Weed Science. 48(1) : 102-104.

Gill, G. S. and Kumar, V. (1969). Weed index: a new method for reporting weeds control traits.Indian Journalof Agronomy. 16 : 9698.

Gomez, K. A. and Gomez, A. A. 1984. Statistical procedure for agricultural research (IInd Edition). John Willey \& Sons Publication : pp : 304-305.

Khan, I. S.; Dubay, W. and Gupta, P. (2014). Taxonomical aspect of coriander. International Journal of Current Research. 6(11) : 9926-9930.

Kondap, S. M. and Upadhaya, U. C. (1985). A practical manual on weed control, oxford and IBH Pub. co., New Delhi, p. 55.

Meena, S. K.; Desai, L. J.; Shaukat Ali and Shivprakashnagar (2013). Effect of weed management on yield, quality and weed parameters in dill seed (Anethum graveolens L.). International Journal of Agricultural Sciences. 9(2) : 723-727.

Meena, S. S. and Mehta, R. S. (2010). Economic feasibility of weed management practices in cumin. Indian Journal of Horticulture. 67:189-192.

Meena, S. S. and Mehta, R. S. (2009). Effect of weed management practices on weed indices, yield and economics of fennel (Foeniculum vulgare Mill.). Indian Journal of Weed Science. 41(3\&4) : 195198.

Meena, S. S.; Kakani, R. K. and Mehta, R. S.
(2009). Economic feasibility of weed management practices in cumin (Cuminum cyminum L.). Journal of Spices and Aromatic Crops. 18(1): 9-12.

Mehriya, M. L.; Yadav, R. S.; Jangir, R. P. and Poonia, B. L. (2008). Effect of different weed management practices on weeds and yield of cumin. Annals of Arid Zone.47(2) : 139-144.

Nagar, R. K and Jain, D. K. (2017). Effect of integrated weed management and balanced fertilization on weed dynamics in coriander (Coriandrum sativum L.). International Journal of Agricultural Science and Research. 7(6) :181-188.

Panse, V. G. and Sukhatme, P. V. (1985). Statistical method for agricultural workers, I.C.A.R., New Delhi.

Patel, S. M.; Amin A. U. and Patel J. A. (2019). Integrated weed management in ajwain (Trachyspermum ammi). International Journal Seed Spices. 9(2) : 37-43.

Patil, J. K.; Amin, A. U.; Tamboli, Y. A. and Patel, U. V. (2020). Growth, yield attributes and yield of coriander (Coriandrum sativum L.) as influenced by weed Management practices and nitrogen levels. International Journal of Current Microbiology and Applied Sciences. 9(4) :328-338.

Sagarka, B. K.; Ramani, B. B.; Mathukia, R. K. and Khanpara, V. D. (2005). Integrated weed management in coriander. Indian Journal of Weed Science. 37(3\&4) : 231233.

Yadav, A.; Patel, J. C.; Mehta, R. S. and Meena, T. (2012) Growth, yields and economics of cumin (Cuminum cyminum L.) production as affected by weed management practices. International Journal Seed Spices. 2(2) : 27-29.

\section{How to cite this article:}

Shilpa Rathod, R. K. Jat, Sanjay Prajapati, Naresh Solanki and Jay Brahmbhatt. 2021. Integrated Weed Management in Coriander (Coriandrum sativum L.). Int.J.Curr.Microbiol.App.Sci. 10(05): 160-169. doi: https://doi.org/10.20546/ijcmas.2021.1005.022 